REVIEW

MECHANISMS IN ENDOCRINOLOGY

\title{
Seizures and type 1 diabetes mellitus: current state of knowledge
}

\author{
Alberto Verrotti, Alessandra Scaparrotta, Cristina Olivieri and Francesco Chiarelli \\ Department of Pediatrics, University of Chieti, Via Dei Vestini 5, 66013 Chieti, Italy \\ (Correspondence should be addressed to F Chiarelli; Email: chiarelli@unich.it)
}

\begin{abstract}
In this review, we will try to analyze the possible coexistence between epilepsy or seizures and type 1 diabetes mellitus (T1DM), in order to establish if there is more than a casual association, and to investigate possible mechanisms underlying this link. Anti-glutamic acid decarboxylase antibodies (GAD-Abs) have been associated with T1DM and a great number of neurological diseases such as epilepsy. Epilepsy can be a feature of a large variety of autoimmune or inflammatory disorders. GAD-Abs can have a role at the basis of the possible link between epilepsy and T1DM, although their real pathogenetic mechanism in neurological diseases is still unknown. Metabolic conditions such as hypoglycemia and hyperglycemia, common problems in diabetic patients, may be also implicated, even if their underlying mechanism is minimally understood.
\end{abstract}

European Journal of Endocrinology 167 749-758

\section{Background}

Type 1 diabetes mellitus (T1DM) is characterized by a chronic, progressive, and immune-mediated destruction of the insulin-producing beta-cells ( $\beta$-cells) in the pancreatic islets of Langerhans. It may occur in association with other autoimmune diseases involving other endocrine tissues, such as thyroid and adrenal glands, or non-endocrine tissue, such as gastric and intestine mucosa (1).

Seizures or epilepsy are observed in many autoimmune or inflammatory disorders, and are linked to the primary disease or secondary to the proinflammatory processes. There could be specific pathogenic antibodies that react with neuronal antigens, causing the central nervous system (CNS) disorder in a subpopulation of patients. Their coexistence may indicate a genetic predisposition common to the two disorders or a simple coincidence (2).

Metabolic abnormalities of diabetes mellitus such as hyperglycemia and hypoglycemia could have a damaging effect on the CNS (3), which may cause seizure; indeed, in endocrine disorders, seizures could be the result of neuroinflammation, autoimmunity, or of metabolic disturbances (2).

Epilepsy is one of the most frequent neurological disorders; the estimated prevalence for active epilepsy ranges from 0.2 to $4.1 \%$ (4). Different causes of epilepsy are commonly being identified, such as structural, metabolic, or genetic causes; however, the majority of epilepsies have unknown etiology (5). Over the last few years, there has been increasing support for the hypothesis that some forms of epilepsy with unknown etiology may have an autoimmune component. Recently, autoantibodies have been found in many CNS disorders often characterized by recurrent seizures (6).

There is increased interest in a possible link between epilepsy and diabetes mellitus. The two conditions have been observed in individuals more frequently than might be expected by chance (7).

This review discusses current understanding of the possible coexistence between seizures or epilepsy and diabetes mellitus in relation to autoimmunity, electroencephalogram (EEG) abnormalities, and glycemic variations, in order to establish if there is more than a casual association, and to investigate possible mechanisms underlying this link.

\section{Epidemiology}

Few epidemiological analyses were performed studying the association between T1DM and epilepsy. There is great interest in the study of McCorry et al. (7), who compared the population prevalence of T1DM in 150000 subjects 15- to 30-year-olds with a cohort of 518 age-matched patients with idiopathic generalized epilepsies (IGEs), founding a greatly significant surplus of T1DM in the IGE cohort, with an odds ratio of 4.4. Successively, O'Connell et al. did not confirm these results, observing that type of epilepsy and prevalence 
in children and adolescents with T1DM are similar to those in the general pediatric population. The prevalence of active epilepsy in the pediatric diabetic population (1384 active patients, aged 0-19 years) of the Diabetes Clinic at the Royal Children's Hospital, Melbourne, Australia was therefore $8.7 / 1000$ or $0.9 \%$ (epilepsy in 15 patients) (8).

Mancardi et al. (9) reviewed 249 T1DM patients (145 male; mean age $16.3 \pm 7.02$ years, mean age at disease onset $7.7 \pm 6.9$ years) attending the Diabetes Clinic, where a collaborative network provides epidemiologic data by an active surveillance model. Six of 249 T1DM patients showed epilepsy. The high proportion of IGE observed (four of $249,1.6 \%$ ) supports the data of McCorry et al. In all patients but one, the epilepsy onset was preceded by T1DM with a mean of 2.8 years. This may reflect different age of onset of the two conditions or alternatively, it could support an autoimmune mechanism starting with T1DM and, then, affecting the CNS (7, 10). On the other hand, it is possible that recurrent episodes of hypoglycemia can result in seizures. In agreement with this hypothesis, Eeg-Olofsson previously demonstrated that in $11 \%$ of children with T1DM an increased prevalence of paroxysmal epileptiform activity compared with $2.7 \%$ of normal controls, noting that marked generalized paroxysmal slow-wave abnormalities were confined to children with recurrent hypoglycemic seizures history $(7,11,12)$.

Epileptic seizures in diabetic patients are mostly reported as focal seizures $(13,14,15,16,17,18,19)$ with or without secondary generalization; epilepsy partialis continua (EPC) $(20,21,22,23,24,25,26$, $27,28)$, occipital seizures $(29,30,31,32,33)$, and reflex seizures $(34,35,36)$ have also been described. However, some cases of generalized epilepsy (childhood absence epilepsy, juvenile absence epilepsy, tonic-clonic seizures on wakening, and juvenile myoclonic epilepsy) associated with T1DM have also been reported $(7,9,37$, 38). From the published cases, it seems that focal seizures are frequently reported as the result of metabolic disturbances, while generalized seizures are mostly linked to autoimmune diseases.

The link between focal seizures and hyperglycemia was first reported in 1965 (14). The phenomenon may not be rare; in a review of 158 cases of nonketotic hyperglycemia (NKH) 19\% had focal motor seizures (15). The focal character of the seizures described during hyperglycemia has been reported consistently over the years $(13,14,16,17,18,19)$. In 1997 Whiting et al. reported the development of intractable focal seizures in three teenagers with previously well-controlled epilepsy. T1DM was diagnosed; glucose levels were high in all patients, with mild ketosis and acidosis only in one patient, and there was prompt cessation of seizures after institution of insulin. The authors concluded that hyperglycemia should be considered in children with epilepsy who develop intractable seizures (19).
EPC is an uncommon, localization-related seizure, characterized by simple focal motor seizures with repetitive clonic jerks, detected in many cortical lesions and metabolic disorders as diabetes mellitus (20). EPC was also observed in the pediatric age group, as a rare manifestation of T1DM (26). Olson et al. (28) described a 6-year-old boy who developed EPC 6 months after diagnosis of T1DM. Lately, Baglietto et al. (27) observed a 3-year-old boy who presented EPC 5 months after T1DM onset, resulting in drug-resistant epilepsy with continuous spike-waves during slow sleep and severe behavioral impairment.

Schober et al., in a very recent study, have estimated the prevalence of epilepsy and possible risk factors in children and adolescents with diabetes mellitus, searching for the concomitant diagnosis of epilepsy or epileptic convulsions and for antiepileptic medication; they have conducted an observational cohort study based on the Diabetes Patienten Verlaufsdokumentation database including data from 45851 patients (52\% male) with T1DM, aged $13.9 \pm 4.3$ years (duration of diabetes mellitus $5.4 \pm 4.2$ years). Seven hundred and five patients with epilepsy were identified, giving a prevalence of 15.5 of 1000 , of which 375 patients treated with antiepileptic therapy and 330 not treated ones. The authors concluded that there is an increased prevalence of epileptic seizures in children and adolescents with diabetes mellitus (39).

The great number of case reports described by international literature increases the enthusiasm for McCorry et al.'s results, supporting the hypothesis that, behind the coexistence of epilepsy and diabetes in the same patient, there is more than a casual association.

Focal neurological deficits associated with hypoglycemia have been frequently observed in diabetic adults, while in children this association was little described. One report presented a series of 19 episodes in seven children with diabetes (40) and another study reported focal motor seizures, followed by ipsilateral hemiparesis associated with hypoglycemia (41).

\section{Autoimmunity as the possible link between epilepsy and T1DM}

Epileptologists are becoming increasingly interested in autoimmune processes and in the search for pathogenic antibodies (42). In particular, anti-glutamic acid decarboxylase antibodies (GAD-Ab) have been associated with T1DM and a large variety of neurological conditions, including epilepsy $(43,44)$. GAD-Ab have been detected in $80 \%$ of patients with new diagnosis of T1DM, in about $80 \%$ of patients with the stiff-man syndrome (SPS) and in some patients with cerebellar ataxia, late-onset autoimmune diabetes mellitus and polyendocrine autoimmunity, palatal myoclonus and epilepsy, and drug-refractory temporal lobe epilepsy (TLE) (44). 
GAD catalyzes the conversion of glutamic acid, the main excitatory amino acid in the brain, to the main inhibitory amino acid gamma-aminobutyric acid (GABA) (2, 45). GABA-secreting neurons and pancreatic beta cells (which may also secrete GABA as a paracrine signal molecule) mostly express GAD (37). Neuronal discharges and seizures may be the result of an imbalance between excitation and inhibition caused by inhibition of GAD activity by autoantibodies $(45,46,47)$.

The full spectrum of neurological syndromes associated with GAD-Ab is not well established. To clarify these issues, Saiz et al. reviewed the clinical and immunological features of patients in whom high GAD-Ab levels were detected in a reference center for T1DM. High levels of GAD-Ab were found in 61 patients; $36 \%$ had SPS, $28 \%$ cerebellar ataxia, $18 \%$ other neurological disorders, and $18 \%$ isolated T1DM. Epilepsy was observed in four (7\%) patients. In one of them, GAD-Ab were measured after the recent onset of an isolated downbeat nystagmus, while in the other three patients, GAD-Ab were detected many years after the onset of the epilepsy, at the time of diagnosis of late-onset T1DM (44). The frequency of high GAD-Ab levels in epileptic patients ranges from 0 to $4 \%(37,44$, 48, 49, 50).

Peltola et al. investigated the prevalence of GAD-Ab in patients with therapy-resistant localizationrelated epilepsy $(n=51)$ and generalized epilepsy $(n=49)$. GAD-Ab were found in eight patients with localization-related epilepsy; high levels of GAD-Ab were observed in two patients, while the remaining six had significantly lower titers, which is a feature of T1DM. The authors concluded that GAD autoimmunity may be associated with refractory localization-related epilepsy (37), although it is important to remember that the majority of epidemiological data suggests that high GAD-Ab levels were found in diabetic patients with IGE. Moreover, there are few studies that detected GAD-Ab in cerebrospinal fluid (CSF). The presence of serum $\mathrm{GAD}-\mathrm{Ab}$ is not a precise marker of intrathecal production of antibodies in these patients; to analyze this aspect, the evaluation of CFS antibodies is more sensitive. The lack of strict correlation between serum GAD-Ab levels and intrathecal synthesis must be taken into consideration when these immunological data are evaluated.

Several studies confirmed elevated GAD-Ab in patients with refractory focal seizures $(27,28,51)$.

In contrast, Kwan et al., measuring serum GAD-Ab in 105 patients with idiopathic or symptomatic epilepsy, did not find any difference in the absolute titer of GAD-Ab between patients with controlled and uncontrolled epilepsy. On the other hand, four uncontrolled epileptic female patients had levels over three times higher than the highest found in the seizure-free group, three of whom were also positive for pancreatic islet cell antibodies (48).

McKnight et al. (49) studied sera obtained from 139 patients and found increased GAD-Ab in five patients
(3.6\%); all of them had drug-resistant epilepsy, onset in childhood or early teens ( $<15$ years), duration of disease for 3 years or greater, and focal abnormalities on EEG. Very high GAD-Ab levels were found in three patients $(2 \%)$ and one of these had hypothyroidism and T1DM.

In another study, GAD-Ab were measured in a series of consecutive unselected epileptic patients observed over a 2-year period; 233 patients (121 women, age range: $6-78)$ were recruited. GAD-Ab were detected in six $(2.58 \%)$ patients; two had IGE associated with T1DM, and the other four patients suffered from cryptogenic temporal epilepsy and no history or signs of T1DM. The authors confirm that GAD autoimmunity may be associated with some forms of epilepsy, with preferential identification in patients with cryptogenic temporal epilepsy (52).

Liimatainen et al. analyzed GAD-Ab in sera of 253 epileptic patients and 200 control subjects, detecting $\mathrm{GAD}-\mathrm{Ab}$ in 15 patients $(5.9 \%)$ and in three control subjects $(1.5 \%)(P=0.026)$. Seven patients $(2.8 \%)$ showed high GAD-Ab titers, six of whom had TLE; low titers were found in all three GAD-Ab-positive control subjects. Intrathecal synthesis of GAD-Ab was observed in two of the five patients with high GAD-Ab titers and available CSF samples; one patient had CSF oligoclonal bands. The prevalence of increased GAD-Ab levels seems to be higher in patients with TLE. The autoimmune origin of the epilepsy is suggested by the immunologic profile of these patients; a positive intrathecal synthesis of GAD-Ab may be an immune response marker (53).

Striano et al. (38) confirmed previous reports, finding the absence of GAD-Ab in isolated IGE and retrospectively identifying two patients with T1DM among their IGE population aged 15 years or older $(n=199)$ with high levels of GAD-Ab.

In another study, investigators selected serum from a GAD-Ab-positive epileptic patient, a GAD-positive diabetic case, two epileptic GAD-Ab-negative patients, and a normal control and registered post-synaptic inhibitory potentials on hippocampal neurons in culture, before and after the application of diluted sera in a patch-clamp study. The frequency of post-synaptic inhibitory potentials significantly increased after application of GAD-positive epileptic serum, while no effect was observed using negative controls' sera: this suggests interference with GABA function and with neuronal inhibition, supporting a pathogenetic role of GAD-Ab in the development of epilepsy (47).

There are few evidences on the pathogenetic role of $\mathrm{GAD}-\mathrm{Ab}$ in neurological diseases. The most important concern is that during GABA exocytosis GAD is never exposed to the outer side of the cell membrane, because it is located in pancreatic beta cells and in neuron cytoplasm. Consequently, it is improbable that GAD-Ab have access from serum and CSF to their target molecule in intact cells, in contrast with the hypothesis 
of in vivo inhibition of GABA synthesis caused by GAD-Ab. On the other hand, antibodies may penetrate cells as often happens with neurons (54). Two mechanisms have been previously suggested for the antibodies-induced suppression of GABA release: reduction of GABA synthesis in the nerve terminal or interference with exocytosis of GABA. Some investigators also assume that GAD-Ab directed to the catalytic site of the molecule may not need to access GAD to display a function because the antibodies could mimic the structure of GABA and cross-react with GABA binding sites accessible on the neuronal surface (54).

Other investigators have demonstrated that circulating antibodies can reach intracellular antigens in two possible ways through the IgG internalization by neurons (55) and through the HSP 70 protein binding, recognized by the immune system making a complex linked to the synaptic vesicle membrane (56). GAD-Ab pathogenicity inside the CNS can be supported by the GAD-Ab intrathecal synthesis, but actually only a few studies have systematically measured the GAD-Ab titer in the CSF of GAD-Ab-positive epileptic patients $(37,46,52,53)$. Intrathecal synthesis of GAD-Ab was calculated in paired serum/CSF samples. Values higher than the IgG index were considered indicators for positive GAD-Ab-specific intrathecal synthesis (44). GAD-Ab may be the biological basis of the association between epilepsy and T1DM (38, 57, 58).

Important antigenic differences between the epitopes recognized by GAD-Ab of diabetic patients and of neurological ones are described by Kim et al. (59), explaining the results' heterogeneity among studies using different GAD-Ab. Low GAD-Ab titers were observed in T1DM patients, while in patients with neurological disorders (cerebellar degeneration or SPS) they may be 25 - to 500 -fold higher $(57,60)$.

As epilepsy often afflicts SPS patients (61), GAD-Ab could have a similar pathogenetic role to that suggested for SPS. Magnetic resonance spectroscopy (MRS) studies in SPS patients (62) demonstrated a significant GABA level decrease in the motor cortex, suggesting that the imbalance of inhibitory and excitatory neurotransmitters equilibrium (with consequent excitatory neurotransmitters predominance) could be secondary to GAD-Ab presence (46). These findings could extend an evidence of the relationship between GAD autoimmunity and seizures, but the implications of this study must be considered with caution because, although interesting, these are the results of only one study.

To test whether the antibodies were associated with changes in cortical GABA levels, Stagg et al. used MRS in four patients with epilepsy and high serum GAD-Ab levels and 10 healthy controls. Patients had significantly lower GABA concentrations within the cortex compared with the control group. The hypothesis that GAD-Ab are markers of a specific disease process with a role for immune-mediated GABAergic dysfunction is supported by the demonstration of an association between high serum GAD-Ab and low cortical GABA levels in patients with epilepsy (45).

Recently, a patient with sudden onset of refractory status epilepticus, in the presence of strong intrathecal GAD-Ab synthesis, was successfully treated with cyclophosphamide (63).

A T-cell-mediated mechanism may be implicated in GAD-Ab-associated neurological disorders, as suggested by the detection of GAD- 65 epitopes by peripheral blood T-cells of patients affected by SPS, considering that T1DM with GAD-Ab positivity is a T-cell-mediated disease (54).

$A$ very recent study observed that the deletion of the genes for IA-2 and IA-2 $\beta$, present in the brain neuroendocrine cells, affected the content and secretion of neurotransmitters and hormones as insulin, FSH, and $\mathrm{LH}$, resulting in a secretion impairment that caused behavioral and learning disturbances, seizures, and reduced life span (64).

Although the real pathogenetic mechanism of GAD$\mathrm{Ab}$ in neurological diseases is still unknown, this extensive analysis of the literature suggests the important role of autoimmunity as the basis of the possible link between epilepsy and T1DM, summarized in the Fig. 1.

\section{Seizures and glycemic variations}

Glucose plays a critical role in brain functions because it represents the main source of metabolic energy generation (65). Focal motor epileptic episodes may be associated with hypoglycemia and $\mathrm{NKH}$; however, these do not occur with ketotic hyperglycemia, probably because of the anticonvulsant action of ketosis (41). Most published reports on diabetic hyperglycemia are concerned with non-ketotic hyperosmolar diabetic coma accompanied by severe hyperglycemia, hyperosmolality, and dehydration, with minimal or no ketoacidosis $(21,66)$, a severe condition which represents one extreme of a biochemical continuum. In practice, actually, diabetics show a spectrum of hyperglycemia and they are often detected before the development of severe hyperosmolarity (18). Although occipital focal seizures have also been described (29, 31 ), the clinical features of focal seizures in NKH are usually those of frontal lobe epilepsy (21). The explanation of this condition by hyperglycemia alone is unsatisfactory, because seizures are rare in diabetic ketoacidosis. It was hypothesized that hyperglycemia leads to a decrease in epileptic seizure threshold by increasing metabolism of GABA and accordingly decreasing the level of GABA, so resulting in a reduction in the seizure threshold $(17,18)$. Another hypothesis involves decrease of seizure threshold due to metabolic disturbance. Hyperosmolality and dehydration associated with hyperglycemia can be considered to be triggers for EPC or other focal seizures, and may lead 


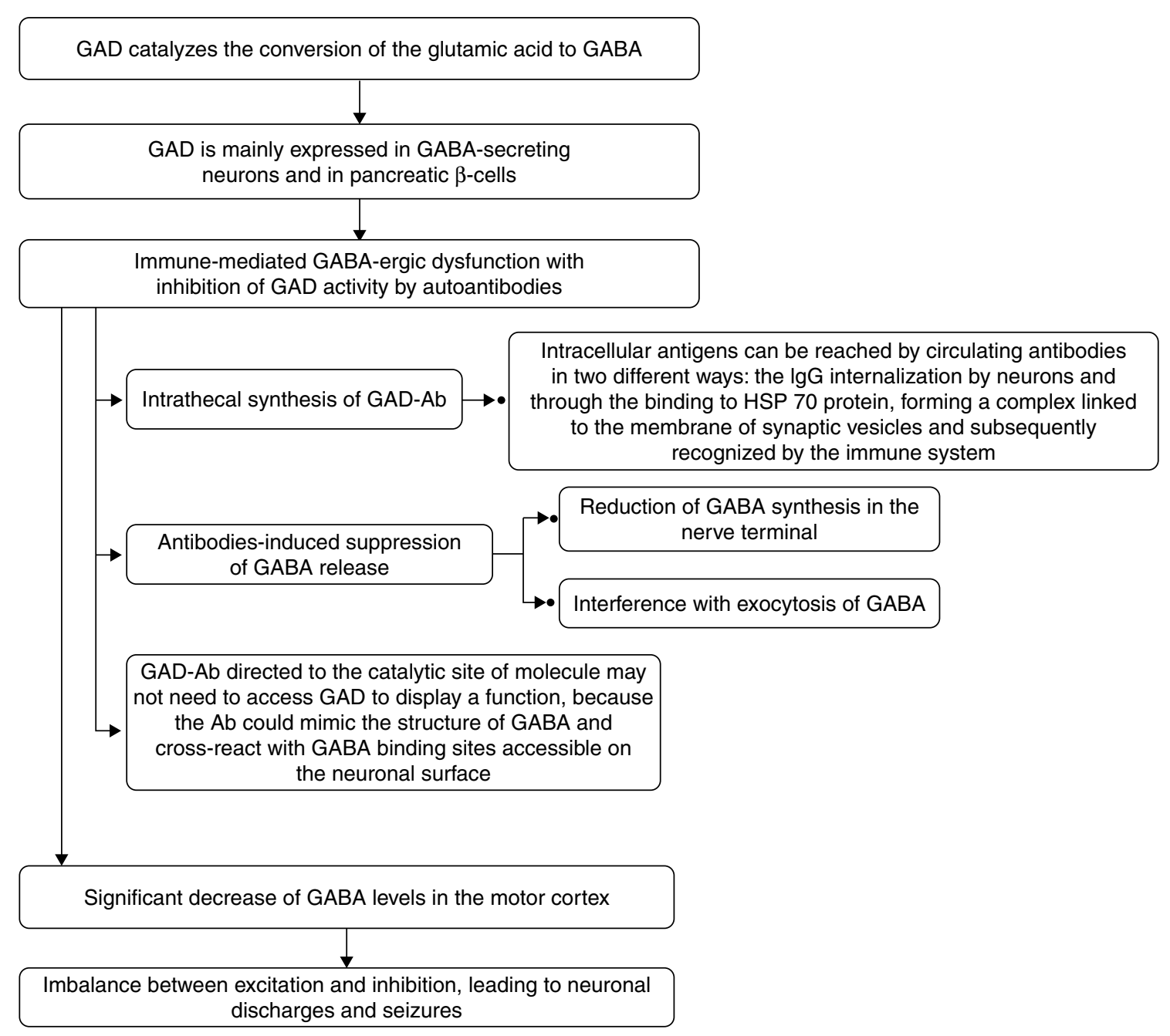

Figure 1 Potential pathogenetic role of GAD-Ab at the basis of the possible link between epilepsy and type 1 diabetes mellitus.

to neurological deficit in some patients (23); in fact, some experimental studies adduced to show that hypertonic solutions will activate existing seizures foci, in particular a potential mechanism is the hyperosmolar irritation of neurons rendered ischemic by an enhanced tendency to vascular disease and by an acute reduction in cerebral blood flow secondary to hyperglycemia and dehydration $(41,67)$. Focal seizures can be symptomatic of structural lesions within the brain; for this reason other authors (18) suggested that a previously existing cortical lesion of ischemic nature might lead to these seizures after acquiring an epileptogenic characteristic under altered metabolic conditions. Some studies $(30,68)$ also described several transient changes in magnetic resonance imaging following seizures. These brain parenchymal changes are typically hyperintense on T2-weighted and fluidattenuated inversion recovery (FLAIR) images. Nevertheless, some patients without evidence of brain damage have been reported $(18,21,23)$. In sum, during hyperglycemia, a focal reduction in blood flow may occur with consequent focal seizures; however, there is no constant relationship between blood glucose levels and the frequency or severity of neurological symptoms (41).

Focal epilepsy may be the first symptom of diabetes in some patients and various studies $(17,18)$ reported cases in which focal seizures can occur in the acute setting of hyperglycemia and can lead to the discovery of diabetes mellitus. While acute hyperglycemia seems to be implicated in the development of frontal lobe epilepsy as EPC (21), prolonged uncontrolled hyperglycemia may be the key to the development of occipital seizures (29), but further studies are needed to clarify how prolonged hyperglycemia contributes to seizures.

As regards ketoacidosis, it has been suggested that ketosis and acidosis have an anticonvulsant effect and therefore a ketogenic diet is thought to be beneficial for epileptic children, although the exact mechanism for 

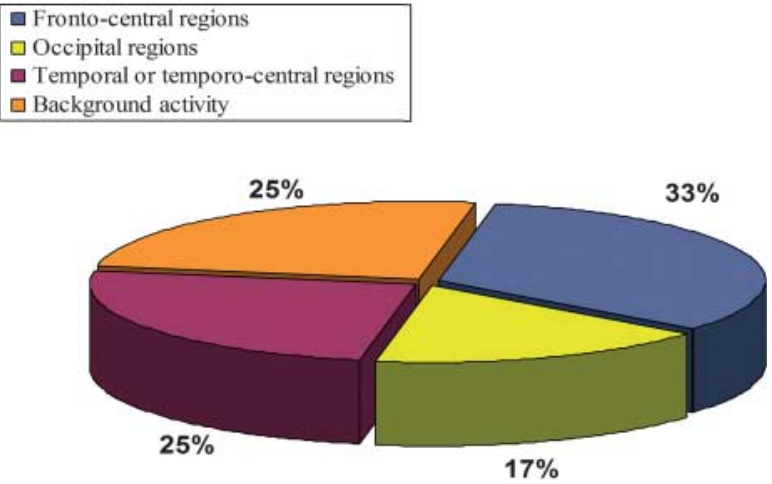

Figure 2 Locations of the most frequent EEG changes consequent to hyperglycemia.

this is still not clearly understood. It has been hypothesized that in this case, intracellular acidosis elevates GABA level by increasing activity of GAD, which leads to a corresponding elevation of seizure threshold $(17,21)$. According to these hypotheses, the presence of seizures in patients with ketoacidosis may indicate the existence of cerebral lesions (69).

However, a very recent study conducted on T1DM children and adolescents without any underlying neurological disease found an association between epilepsy and diabetic ketoacidosis, observing almost double the risk for diabetic ketoacidosis in patients with epilepsy compared with T1DM alone ones (39).

Hypoglycemia is a common problem in diabetic patients treated with insulin and can induce various neurological disturbances, such as confusion, motor restlessness, hypotonia, and epileptic seizures, which represent a relatively common acute symptom $(41,70)$. The association between hypoglycemia and epilepsy or seizures has not yet been fully understood. A significantly low glucose level can alter cortical excitability so determining a disproportion between excitation and inhibition. Indeed, several experimental studies have shown that the loss of high-energy substrates leads to the release of excitatory amino acids that result in hyperexcitability and consequent excitotoxicity (71). These effects may be more pronounced in some brain regions than in others, the temporal lobe and hippocampus appearing to be particularly susceptible (72). Although these data provide a convincing explanation for the possible occurrence of focal epileptic events associated with hypoglycemia, this seizure type has rarely been reported and documented, while generalized tonic-clonic results are the most common $(41,65,73)$. Furthermore, according to some authors, it is clear that the neurological deficits could not be related only to the hypoglycemic episode, because in a few episodes, administration of sugar did not result in immediate reversal of the neurological deficit $(40,41)$.

Since hypoglycemia occurs frequently in children with diabetes, it can be postulated that generalized and focal neurological deficits are much more common than reported in the literature, and at times such a deficit may be the first clinical symptom of diabetes in the pediatric age group (41).

\section{EEG abnormalities and glycemic control}

Patients with T1DM can show EEG abnormalities (74, $75,76,77)$. There are conflicting opinions regarding the role of diabetic ketoacidosis (DKA) in the development of seizures: however, the first report of EEG changes in children with DKA or ketosis dates back to 1981 (78). Several studies reported the association between severe hypoglycemia (SH) and EEG abnormalities in diabetic children and adolescents $(3,74,76,79$, 80 ). Haumont et al. described one of the first reports of a significant positive correlation between EEG abnormalities and degree of diabetic control, but no definite increase was noted in relation to the duration of diabetes $(79,80)$. In 1983, 270 juvenile children from the Montreal Children's Hospital Diabetic Clinic were reviewed. Numbers of epileptiform EEG abnormalities were significantly increased in the initial EEG of diabetic patients who later had a hypoglycemic convulsion (81). In the same year, Halonen et al. (82) recorded 498 EEGs from 195 diabetic children; the children were divided into stable and labile groups according to diabetes control. The labile children had significantly more abnormal EEGs than the stable children. In 1989, Soltész \& Acsádi (3) described the EEG abnormalities in 70 diabetic children and 70 normal controls. Twentysix percent had abnormal electroencephalograms, while these abnormalities were found in only $7 \%$ of the 70 healthy children; moreover, about $80 \%$ of the children with previous SH presented abnormal EEG, compared with only $30 \%$ in the non-hypoglycemic group. In another study (75), quantitative EEGs of 44 insulin-dependent diabetic patients were compared with normal controls, and a correlation between high values of $\mathrm{HbA} 1 \mathrm{c}$ and decreased relative power of the alpha

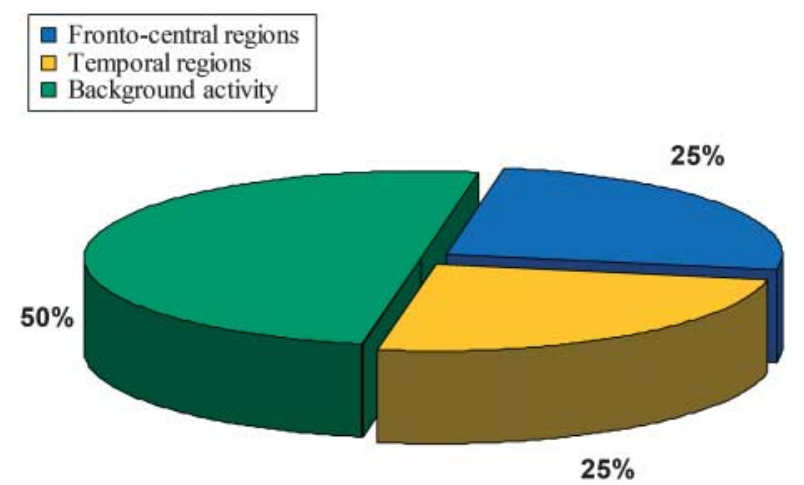

Figure 3 Locations of the most frequent EEG changes consequent to hypoglycemia. 
band was found. Patients with previous ketoacidotic episodes had increased relative power of the delta/theta band and decreased relative power of the alpha band. Patients with $\mathrm{HbA} 1 \mathrm{c}$ values $<8.5 \%$ exhibited slowing of background activity compared with normal controls. Other detailed EEG descriptions were reported in the following years (Fig. 2) (76, 77, 83, 84, 85). Figures 2 and 3 show the most frequent cerebral sites affected during episodes of hyperglycemia and hypoglycemia respectively.

Two more recent studies were performed in 2005 (74) and in 2011 (86). In the first study, EEG abnormalities were observed in T1DM adolescents treated with multiple insulin injection therapy. Decrease of the power of the faster frequencies (alpha, beta, and gamma) were most pronounced in the temporal regions, while increased power of the slower frequencies (theta and delta), and a reduction of the alpha peak frequency were found in the frontal regions. Strong correlations were found in the increase in theta activity with a history of SH, the increase in delta activity with high acute $\mathrm{HbA} 1 \mathrm{c}$ levels, and the decrease in alpha peak frequency with high long-term HbAlc levels $(74,86)$. In the second work, the results of a precedent study (76) were compared with the results of a 16-year follow-up of the same patients involved in the first study in order to assess whether those diabetic patients with childhood exposure to SH have EEG abnormalities in adulthood (86). Participants with prior $\mathrm{SH}$, when investigated at about 13 years of age, presented reduced alpha activity and increased fronto-central theta activity (76). However, childhood SH seems not to be associated with persistent EEG abnormalities after 16 years of follow-up; therefore, impaired learning conditions in childhood (87), rather than cerebral damage caused by $\mathrm{SH}$, may explain the association of $\mathrm{SH}$ in childhood with reduced adulthood cognition (86).

In summary, EEG changes can be common in children with diabetes. These changes may persist in some cases, possibly accounting for the increased frequency of EEG abnormalities in diabetic children (78). In spite of the occurrence of many EEG abnormalities, it is unclear if there is a link between these EEG findings and the later development of seizures.

\section{Conclusions}

Epilepsy can be a feature of diabetic patients. The available evidence suggests that specific pathogenic antibodies can react with neuronal antigens in a subpopulation of diabetic patients. GAD-Ab can be at the basis of the possible link between epilepsy and T1DM, although there are questions regarding the relative role of these antibodies. In fact, the pathogenicity of these antibodies needs to be clarified to determine if they are causative or simply a marker of underlying inflammatory processes. Efforts should be applied to identifying new antibodies which could help to better understand the link between epilepsy and T1DM. Further studies are needed to look for GAD-Ab and other autoantibodies in additional cohorts and to define any clinical phenotype that might be associated with these autoantibodies. Moreover, it is likely that both metabolic extremes, SH and severe hyperglycemia, may contribute to the development of epilepsy in patients with T1DM.

Diabetic patients need a multidisciplinary approach that involves pediatric endocrinologists and neurologists in order to optimize therapeutic management and follow-up.

\section{Declaration of interest}

The authors declare that there is no conflict of interest that could be perceived as prejudicing the impartiality of the review reported.

\section{Funding}

This review did not receive any specific grant from any funding agency in the public, commercial or not-for-profit sector.

\section{References}

1 Tsirogianni A, Pipi E \& Soufleros K. Specificity of islet cell autoantibodies and coexistence with other organ specific autoantibodies in type 1 diabetes mellitus. Autoimmunity Reviews 2009 8 687-691. (doi:10.1016/j.autrev.2009.02.019)

2 Vincent A \& Crino PB. Systemic and neurologic autoimmune disorders associated with seizures or epilepsy. Epilepsia 201152 12-17. (doi:10.1111/j.1528-1167.2011.03030.x)

3 Soltész G \& Acsádi G. Association between diabetes, severe hypoglycaemia, and electroencephalographic abnormalities. Archives of Disease in Childhood 198964 992-996. (doi:10.1136/adc.64. 7.992)

4 Ottman R, Lipton RB, Ettinger AB, Cramer JA, Reed ML, Morrison A \& Wan GJ. Comorbidities of epilepsy: results from the Epilepsy Comorbidities and Health (EPIC) survey. Epilepsia 2011 52 308-315. (doi:10.1111/j.1528-1167.2010.02927.x)

5 Irani SR, Bien CG \& Lang B. Autoimmune epilepsies. Current Opinion in Neurology 201124 146-153. (doi:10.1097/WCO. Ob013e3283446fo5)

6 Bien CG \& Scheffer IE. Autoantibodies and epilepsy. Epilepsia 2011 52 18-22. (doi:10.1111/j.1528-1167.2011.03031.x)

7 McCorry D, Nicolson A, Smith D, Marson A, Feltbower RG \& Chadwick DW. An association between type 1 diabetes and idiopathic generalized epilepsy. Annals of Neurology $2006 \mathbf{5 9}$ 204-206. (doi:10.1002/ana.20727)

8 O'Connell MA, Harvey AS, Mackay MT \& Cameron FJ. Does epilepsy occur more frequently in children with Type 1 diabetes? Journal of Paediatrics and Child Health 200844 586-589. (doi:10.1111/j.1440-1754.2008.01387.x)

9 Cotellessa M, Barbieri P, Mazzella M, Bonassi S, Minicucci L \& Lorini R. High incidence of childhood type 1 diabetes in Liguria, Italy, from 1989 to 1998. Diabetes Care 200326 1786-1789. (doi:10.2337/diacare.26.6.1786)

10 Mancardi MM, Striano P, Giannattasio A, Baglietto MG, Errichiello L, Zara F, Prato G, Minuto N, Veneselli E, Lorini R \& D'Annunzio G. Type 1 diabetes and epilepsy: more than a casual association? Epilepsia 201051 320-321. (doi:10.1111/j.15281167.2009.02380.x) 
11 Eeg-Olofsson O \& Petersen I. Childhood diabetic neuropathy. A clinical and neurophysiological study. Acta Paediatrica Scandinavica 196955 163-176. (doi:10.1111/j.1651-2227.1966.tb15222.x)

12 Eeg-Olofsson O. Hypoglycemia and neurological disturbances in children with diabetes mellitus. Acta Paediatrica Scandinavica 1977 270 91-96. (doi:10.1111/j.1651-2227.1977.tb15127.x)

13 Huang CW, Hsieh YJ, Huang CC \& Tsai JJ. Non-ketotic hyperglycaemia-related paroxysmal bilateral hand paraesthesia misdiagnosed as diabetic neuropathy. Neurological Sciences 2006 27 180-182. (doi:10.1007/s10072-006-0665-0)

14 Maccario M, Messis CP \& Vastola F. Focal seizures as a manifestation of hyperglycaemia without ketoacidosis. Neurology 196515 195-206. (doi:10.1212/WNL.15.3.195)

15 Loeb JH. The hyperosmolar state. New England Journal of Medicine 1974290 1184-1187. (doi:10.1056/NEJM19740523290 2107)

16 DeCaro LJ, Reinig M \& Croft BT. Clonic focal seizure of the foot secondary to nonketotic hyperglycemia. Journal of the American Podiatric Medical Association 200292 109-111.

17 Hennis A, Corbin D \& Fraser H. Focal seizures and non-ketotic hyperglycaemia. Journal of Neurology, Neurosurgery, and Psychiatry 199255 195-197. (doi:10.1136/jnnp.55.3.195)

18 Grant C \& Warlow C. Focal epilepsy in diabetic non-ketotic hyperglycaemia. BMJ 1985290 1204-1205. (doi:10.1136/bmj. 290.6476.1204)

19 Whiting S, Camfield P, Arab D \& Salisbury S. Insulin-dependent diabetes mellitus presenting in children as frequent, medically unresponsive, partial seizures. Journal of Child Neurology 199712 178-180. (doi:10.1177/088307389701200305)

20 Mukherjee V, Mukherjee A, Mukherjee A \& Halder A. Type 1 diabetes mellitus in a child presenting with epilepsy partialis continua. Journal of the Indian Medical Association $2007 \mathbf{1 0 5}$ 340-342.

21 Cokar O, Aydin B \& Ozer F. Non-ketotic hyperglycaemia presenting as epilepsia partialis continua. Seizure 200413 264-269. (doi:10.1016/S1059-1311(03)00155-9)

22 Guerreiro CA, Falcão AE \& Silveira DC. Focal seizures in nonketotic hyperglycemia. Arquivos de Neuro-Psiquiatria 199149 447-449. (doi:10.1590/S0004-282X1991000400014)

23 Singh BM \& Strobos RJ. Epilepsia partialis continua associated with nonketotic hyperglycemia: clinical and biochemical profile of 21 patients. Annals of Neurology 19808 155-160. (doi:10.1002/ ana.410080205)

24 Sabitha KM, Girija AS \& Vargese KS. Seizures in hyperglycemic patients. Journal of the Association of Physicians of India 200149 723-726.

25 Kumar S. Epilepsia partialis continua stopped by insulin. Journal of the Royal Society of Medicine 200497 332-333. (doi:10.1258/ jrsm.97.7.332)

26 Sabharwal RK, Gupta M, Sharma D \& Puri V. Juvenile diabetes manifesting as epilepsia partialis continua. Journal of the Association of Physicians of India 198937 603-604.

27 Baglietto MG, Mancardi MM, Giannattasio A, Minuto N, Rossi A, Capovilla G, Veneselli E, Lorini R \& D’Annunzio G. Epilepsia partialis continua in type 1 diabetes: evolution into epileptic encephalopathy with continuous spike-waves during slow sleep. Neurological Sciences 200930 509-512. (doi:10.1007/s10072009-0122-y)

28 Olson JA, Olson DM, Sandborg C, Alexander S \& Buckingham B. Type 1 diabetes mellitus and epilepsia partialis continua in a 6-year-old boy with elevated anti-GAD65 antibodies. Pediatrics 2002109 E50. (doi:10.1542/peds.109.3.e50)

29 Hung WL, Hsieh PF, Lee YC \& Chang MH. Occipital lobe seizures related to marked elevation of hemoglobin A1C: report of two cases. Seizure 201019 359-362. (doi:10.1016/j.seizure.2010. 05.006)

30 Raghavendra S, Ashalatha R, Thomas SV \& Kesavadas C. Focal neuronal loss, reversible subcortical focal $\mathrm{T} 2$ hypointensity in seizures with a nonketotic hyperglycemic hyperosmolar state. Neuroradiology 200749 299-305. (doi:10.1007/s00234-0060189-6)
31 Wang CP, Hsieh PF, Chen CC, Lin WY, Hu WH, Yang DY \& Chang MH. Hyperglycemia with occipital seizures: images and visual evoked potentials. Epilepsia $2005 \mathbf{4 6}$ 1140-1144. (doi:10.1111/j.1528-1167.2005.56404.x)

32 Martínez MD \& Megías SM. Occipital seizures with electroencephalographic alterations as the initial manifestation of diabetes mellitus. Endocrinología y Nutrición 200956 458-460. (doi:10.1016/S1575-0922(09)73314-5)

33 Moien-Afshari F \& Téllez-Zenteno JF. Occipital seizures induced by hyperglicemia: a case report and review of literature. Seizure 2009 18 382-385. (doi:10.1016/j.seizure.2008.12.001)

34 Tiras R, Mutlu A, Ozben S, Aydemir T \& Ozer F. Forced eye closureinduced reflex seizure and non-ketotic hyperglycemia. Annals of Saudi Medicine 200929 313-315. (doi:10.4103/0256-4947. 55319)

35 Siddiqi ZA, VanLandingham KE \& Husain AM. Reflex seizures and non-ketotic hyperglycemia: an unresolved issue. Seizure 200211 63-66. (doi:10.1053/seiz.2001.0559)

36 Ozer F, Mutlu A \& Ozkayran T. Reflex epilepsy and non-ketotic hyperglycemia. Epileptic Disorders: International Epilepsy Journal with Videotape 20035 165-168.

37 Peltola J, Kulmala P, Isojärvi J, Saiz A, Latvala K, Palmio J, Savola K, Knip M, Keränen T \& Graus F. Autoantibodies to glutamic acid decarboxylase in patients with therapy-resistant epilepsy. Neurology 200055 46-50. (doi:10.1212/WNL.55.1.46)

38 Striano P, Perruolo G, Errichiello L, Formisano P, Beguinot F, Zara F \& Striano S. Glutamic acid decarboxylase antibodies in idiopathic generalized epilepsy and type 1 diabetes. Annals of Neurology 200863 127-128. (doi:10.1002/ana.21011)

39 Schober E, Otto KP, Dost A, Jorch N \& Holl R. German/Austrian DPV, initiative and the BMBF competence network diabetes, association of epilepsy and type 1 diabetes mellitus in children and adolescents: is there an increased risk for diabetic ketoacidosis? Journal of Pediatrics 2012160 662-666. (doi:10.1016/j.jpeds. 2011.09.054)

40 Wayne EA, Dean HJ, Booth F \& Tenenbein M. Focal neurological deficits associated with hypoglycemia in children with diabetes. Journal of Pediatrics 1990117 575-577. (doi:10.1016/SO0223476(05)80691-2)

41 Lahat E, Barr J \& Bistritzer T. Focal epileptic episodes associated with hypoglycemia in children with diabetes. Clinical Neurology and Neurosurgery 199597 314-316. (doi:10.1016/0303-8467(95) 00072-R)

42 Ranua J, Luoma K, Auvinen A, Haapala AM, Mäki M, Peltola J, Raitanen J \& Isojärvi JI. Antimitochondrial antibodies in patients with epilepsy. Epilepsy E Behavior 2005 7 95-97. (doi:10.1016/j. yebeh.2005.04.001)

43 Striano P, Errichiello L \& Striano S. Autoantibodies to glutamic acid decarboxylase in patients with epilepsy: what is their clinical relevance? Epilepsy \& Behavior 201120145.

44 Saiz A, Blanco Y, Sabater L, González F, Bataller L, Casamitjana R, Ramió-Torrentà L \& Graus F. Spectrum of neurological syndromes associated with glutamic acid decarboxylase antibodies: diagnostic clues for this association. Brain $2008 \mathbf{1 3 1} 2553-2563$. (doi:10.1093/brain/awn183)

45 Stagg CJ, Lang B, Best JG, McKnight K, Cavey A, Johansen-Berg H, Vincent A \& Palace J. Autoantibodies to glutamic acid decarboxylase in patients with epilepsy are associated with low cortical GABA levels. Epilepsia $2010 \mathbf{5 1}$ 1898-1901. (doi:10.1111/j. 1528-1167.2010.02644.x)

46 Errichiello L, Striano S, Zara F \& Striano P. Temporal lobe epilepsy and anti glutamic acid decarboxylase autoimmunity. Neurological Sciences 2011 32 547-550. (doi:10.1007/s10072-011-0566-8)

47 Vianello M, Bisson G, Dal Maschio M, Vassanelli S, Girardi S, Mucignat C, Fountzoulas K \& Giometto B. Increased spontaneous activity of a network of hippocampal neurons in culture caused by suppression of inhibitory potentials mediated by anti-gad antibodies. Autoimmunity $2008 \quad 41$ 66-73. (doi:10.1080/ $08916930701619565)$ 
48 Kwan P, Sills GJ, Kelly K, Butler E \& Brodie MJ. Glutamic acid decarboxylase autoantibodies in controlled and uncontrolled epilepsy: a pilot study. Epilepsy Research 200042 191-195. (doi:10.1016/S0920-1211(00)00180-7)

49 McKnight K, Jiang Y, Hart Y, Cavey A, Wroe S, Blank M, Shoenfeld Y, Vincent A, Palace J \& Lang B. Serum antibodies in epilepsy and seizure-associated disorders. Neurology $2005 \mathbf{6 5}$ 1730-1736. (doi:10.1212/01.wnl.0000187129.66353.13)

50 Verrotti A, Greco R, Altobelli E, Latini G, Morgese G \& Chiarelli F. Anticardiolipin, glutamic acid decarboxylase, and antinuclear antibodies in epileptic patients. Clinical and Experimental Medicine 20033 32-36. (doi:10.1007/s102380300013)

51 Yoshimoto T, Doi M, Fukai N, Izumiyama H, Wago T, Minami I, Uchimura I \& Hirata Y. Type 1 diabetes mellitus and drug-resistant epilepsy: presence of high titer of anti-glutamic acid decarboxylase autoantibodies in serum and cerebrospinal fluid. Internal Medicine 200544 1174-1177. (doi:10.2169/internalmedicine.44.1174)

52 Errichiello L, Perruolo G, Pascarella A, Formisano P, Minetti C, Striano S, Zara F \& Striano P. Autoantibodies to glutamic acid decarboxylase (GAD) in focal and generalized epilepsy: a study on 233 patients. Journal of Neuroimmunology 2009211 120-123. (doi:10.1016/j.jneuroim.2009.04.010)

53 Liimatainen S, Peltola M, Sabater L, Fallah M, Kharazmi E, Haapala AM, Dastidar P, Knip M, Saiz A \& Peltola J. Clinical significance of glutamic acid decarboxylase antibodies in patients with epilepsy. Epilepsia 201051 760-767. (doi:10.1111/j.15281167.2009.02325.x)

54 Vianello M, Tavolato B \& Giometto B. Glutamic acid decarboxylase autoantibodies and neurological disorders. Neurological Sciences 200223 145-151. (doi:10.1007/s100720200055)

55 Fishman PS \& Drachman DB. Internalization of $\mathrm{IgG}$ in motoneurons of patients with ALS: selective or nonselective? Neurology 199545 1551-1554. (doi:10.1212/WNL.45.8.1551)

56 Jin H, Wu H, Osterhaus G, Wei J, Davis K, Sha D, Floor E, Hsu CC, Kopke RD \& Wu JY. Demonstration of functional coupling between gamma -aminobutyric acid (GABA) synthesis and vesicular GABA transport into synaptic vesicles. PNAS 2003100 4293-4298. (doi:10.1073/pnas.0730698100)

57 Rüegg S. Anti-glutamic acid decarboxylase anti-bodies: the missing link between epilepsy and diabetes. Annals of Neurology $200659728-729$.

58 Vulliemoz S \& Seeck M. An association between type 1 diabetes and idiopathic generalized epilepsy. Annals of Neurology $2006 \mathbf{5 9}$ 728. (doi:10.1002/ana.20830)

59 Kim J, Namchuk M, Bugawan T, Fu Q, Jaffe M, Shi Y, Aanstoot HJ, Turck CW, Erlich H, Lennon V \& Baekkeskov S. Higher autoantibody levels and recognition of a linear NH2-terminal epitope in the autoantigen GAD65, distinguish stiff-man syndrome from insulin-dependent diabetes mellitus. Journal of Experimental Medicine 1994180 595-606. (doi:10.1084/jem. $180.2 .595)$

60 Honorat J, Saiz A, Giometto B, Vincent A, Brieva L, de Andres C, Maestre J, Fabien N, Vighetto A, Casamitjana R, Thivolet C, Tavolato B, Antoine J, Trouillas P \& Graus F. Cerebellar ataxia with antiglutamic acid decarboxylase antibodies. Archives of Neurology 200158 225-230. (doi:10.1001/archneur. 58.2.225)

61 Espay AJ \& Chen R. Rigidity and spasms from autoimmune encephalomyelopathies: stiff-person syndrome. Muscle $\mathcal{E}$ Nerve 200634 677-690. (doi:10.1002/mus.20653)

62 Levy LM, Levy-Reis I, Fujii M \& Dalakas MC. Brain gammaaminobutyric acid changes in stiff-person syndrome. Archives of Neurology 200562 970-974. (doi:10.1001/archneur.62.6.970)

63 Kanter IC, Huttner HB, Staykov D, Biermann T, Struffert T, Kerling F, Hilz MJ, Schellinger PD, Schwab S \& Bardutzky J. Cyclophosphamide for anti-GAD antibody-positive refractory status epilepticus. Epilepsia 200849 914-920. (doi:10.1111/j. 1528-1167.2007.01500.x)

64 Nishimura T, Kubosaki A, Ito Y \& Notkins AL. Disturbances in the secretion of neurotransmitters in IA-2/IA-2 $\beta$ null mice: changes in behavior, learning and life span. Neuroscience 2009 159 427-437. (doi:10.1016/j.neuroscience.2009.01.022)
65 Lapenta L, Di Bonaventura C, Fattouch J, Bonini F, Petrucci S, Gagliardi S, Casciato S, Manfredi M, Prencipe M \& Giallonardo AT. Focal epileptic seizure induced by transient hypoglycaemia in insulin-treated diabetes. Epileptic Disorders: International Epilepsy Journal with Videotape 201012 84-87.

66 Windebank AJ \& McEvoy KM. Diabetes and the nervous system. In Neurology and General Medicine: The Neurological Aspect of Medical Disorders, edn 2, pp 349-381. Ed. MJ Aminoff, New York: Churchill Livingstone, 1995.

67 Manford M, Fuller GN \& Wade JP. "Silent diabetes": non-ketotic hyperglycaemia presenting as aphasic status epilepticus. Journal of Neurology, Neurosurgery, and Psychiatry $1995 \quad 59 \quad 99-100$. (doi:10.1136/jnnp.59.1.99-a)

68 Goto H, Kumagai T \& Momozaki N. MRI findings of occipital seizures in non-ketotic hyperglycemia. Internal Medicine 201150 367-368. (doi:10.2169/internalmedicine.50.4684)

69 Lee HS \& Hwang JS. Cerebral infarction associated with transient visual loss in child with diabetic ketoacidosis. Diabetic Medicine 201128 516-518. (doi:10.1111/j.1464-5491.2011.03241.x)

70 Varghese P, Gleason V, Sorokin R, Senholzi C, Jabbour S \& Gottlieb JE. Hypoglycemia in hospitalized patients treated with antihyperglycemic agents. Journal of Hospital Medicine $2007 \mathbf{4}$ 234-240. (doi:10.1002/jhm.212)

71 McCall AL. Cerebral glucose metabolism in diabetes mellitus. European Journal of Pharmacology 2004490 147-158. (doi:10. 1016/j.ejphar.2004.02.052)

72 Auer RN. Hypoglycemic brain damage. Metabolic Brain Disease 2004 19 169-175. (doi:10.1023/B:MEBR.0000043967.78763.5b)

73 Caraballo RH, Sakr D, Mozzi M, Guerrero A, Adi JN, Cersósimo RO \& Fejerman N. Symptomatic occipital lobe epilepsy following neonatal hypoglycaemia. Pediatric Neurology 200431 24-29. (doi:10.1016/j.pediatrneurol.2003.12.008)

74 Hyllienmark L, Maltez J, Dandenell A, Ludvigsson J \& Brismar T. EEG abnormalities with and without relation to severe hypoglycaemia in adolescents with type 1 diabetes. Diabetologia $2005 \mathbf{4 8}$ 412-419. (doi:10.1007/s00125-004-1666-2)

75 Hauser E, Strohmayer C, Seidl R, Birnbacher R, Lischka A \& Schober EJ. Quantitative EEG in young diabetics. Journal of Child Neurology 199510 330-334. (doi:10.1177/08830 7389501000419)

76 Bjorgaas M, Sand T \& Gimse R. Quantitative EEG in type 1 diabetic children with and without episodes of severe hypoglycemia: a controlled, blind study. Acta Neurologica Scandinavica 199693 398-402. (doi:10.1111/j.1600-0404.1996.tb00017.x)

77 Howorka K, Pumprla J, Saletu B, Anderer P, Krieger M \& Schabmann A. Decrease of vigilance assessed by EEG-mapping in type I diabetic patients with history of recurrent severe hypoglycaemia. Psychoneuroendocrinology $2000 \quad 25 \quad 85-105$. (doi:10.1016/S0306-4530(99)00041-4)

78 Tsalikian E, Becker DJ, Crumrine PK, Daneman D \& Drash AL. Electroencephalographic changes in diabetic ketosis in children with newly and previously diagnosed insulin-dependent diabetes mellitus. Journal of Pediatrics 198199 355-359. (doi:10.1016/ S0022-3476(81)80317-4)

79 Haumont D, Dorchy H \& Pelc S. EEG abnormalities in diabetic children: influence of hypoglycemia and vascular complications. Clinical Pediatrics $1979 \quad \mathbf{1 8} \quad 750-753 . \quad$ (doi:10.1177/ 000992287901801205)

80 Dorchy H. To: Hyllienmark L, Maltez J, Dandenell A, Ludvigsson J \& Brismar T. EEG abnormalities with and without relation to severe hypoglycaemia in adolescents with type 1 diabetes. Diabetologia 200548 2191-2192 (Comment on Diabetologia $200548412-419)$.

81 Keene DL, Metrakos K, Belmonte M, Watters GV \& Singer S. EEG abnormalities and convulsions in juvenile diabetes mellitus. Canadian Journal of Neurological Sciences 198310 198-199.

82 Halonen H, Hiekkala H, Huupponen T \& Häkkinen VK. A followup EEG study in diabetic children. Annals of Clinical Research 1983 15 167-172. 
83 Bjørgaas M, Sand T, Vik T \& Jorde R. Quantitative EEG, during controlled hypoglycaemia in diabetic and non-diabetic children. Diabetic Medicine 199815 30-37. (doi:10.1002/(SICI)10969136(199801)15:1<30::AID-DIA526> 3.0.CO;2-R)

84 Tupola S, Saar P \& Rajantie J. Abnormal electroencephalogram at diagnosis of insulin-dependent diabetes mellitus may predict severe symptoms of hypoglycemia in children. Journal of Pediatrics 1998133 792-794. (doi:10.1016/S0022-3476(98)70154-4)

85 Brismar T, Hyllienmark L, Ekberg K \& Johansson BL. Loss of temporal lobe beta power in young adults with type 1 diabetes mellitus. Neuroreport $2002 \quad 13 \quad 2469-2473$. (doi:10.1097/ 00001756-200212200-00019)
86 Åsvold BO, Sand T, Hestad KA \& Bjørgaas MR. Quantitative EEG in type 1 diabetic adults with childhood exposure to severe hypoglycaemia: a 16 year follow-up study. Diabetologia $2011 \mathbf{5 4}$ 2404-2408. (doi:10.1007/s00125-011-2208-3)

87 Northam EA \& Lin A. Hypoglycaemia in childhood onset type 1 diabetes-part villain, but not the only one. Pediatric Diabetes 2010 11 134-141. (doi:10.1111/j.1399-5448.2009.00545.x)

Received 2 May 2012

Accepted 6 September 2012 